\title{
Dying with SARS-CoV-2 infection-an autopsy study of the first consecutive 80 cases in Hamburg, Germany
}

\author{
Carolin Edler $^{1}$ • Ann Sophie Schröder ${ }^{1} \cdot$ Martin Aepfelbacher $^{2}$ - Antonia Fitzek ${ }^{1}$ - Axel Heinemann ${ }^{1} \cdot$ Fabian Heinrich $^{1}$. \\ Anke Klein ${ }^{1}$ - Felicia Langenwalder ${ }^{1}$. Marc Lütgehetmann ${ }^{3}$ • Kira Meißner ${ }^{1}$ - Klaus Püschel ${ }^{1}$ - Julia Schädler ${ }^{1}$. \\ Stefan Steurer ${ }^{2} \cdot$ Herbert Mushumba $^{1} \cdot$ Jan-Peter Sperhake ${ }^{1}$
}

Received: 1 May 2020 / Accepted: 15 May 2020/Published online: 4 June 2020

(C) Springer-Verlag GmbH Germany, part of Springer Nature 2020

\begin{abstract}
Autopsies of deceased with a confirmed severe acute respiratory syndrome coronavirus 2 (SARS-CoV-2) infection can provide important insights into the novel disease and its course. Furthermore, autopsies are essential for the correct statistical recording of the coronavirus disease 2019 (COVID-19) deaths. In the northern German Federal State of Hamburg, all deaths of Hamburg citizens with ante- or postmortem PCR-confirmed SARS-CoV-2 infection have been autopsied since the outbreak of the pandemic in Germany. Our evaluation provides a systematic overview of the first 80 consecutive full autopsies. A proposal for the categorisation of deaths with SARS-CoV-2 infection is presented (category 1: definite COVID-19 death; category 2: probable COVID-19 death; category 3: possible COVID-19 death with an equal alternative cause of death; category 4: SARSCoV-2 detection with cause of death not associated to COVID-19). In six cases, SARS-CoV-2 infection was diagnosed postmortem by a positive PCR test in a nasopharyngeal or lung tissue swab. In the other 74 cases, SARS-CoV-2 infection had already been known antemortem. The deceased were aged between 52 and 96 years (average 79.2 years, median 82.4 years). In the study cohort, 34 deceased were female (38\%) and 46 male (62\%). Overall, $38 \%$ of the deceased were overweight or obese. All deceased, except for two women, in whom no significant pre-existing conditions were found autoptically, had relevant comorbidities (in descending order of frequency): (1) diseases of the cardiovascular system, (2) lung diseases, (3) central nervous system diseases, (4) kidney diseases, and (5) diabetes mellitus. A total of 76 cases (95\%) were classified as COVID-19 deaths, corresponding to categories 1-3. Four deaths (5\%) were defined as non-COVID-19 deaths with virus-independent causes of death. In eight cases, pneumonia was combined with a fulminant pulmonary artery embolism. Peripheral pulmonary artery embolisms were found in nine other cases. Overall, deep vein thrombosis has been found in $40 \%$ of the cases. This study provides the largest overview of autopsies of SARS-CoV-2-infected patients presented so far.
\end{abstract}

Keywords Coronavirus $\cdot$ SARS-CoV-2 $\cdot$ COVID-19 $\cdot$ Autopsy $\cdot$ Pulmonary embolism $\cdot$ Venous thromboembolic disease

Carolin Edler, Ann Sophie Schröder, Herbert Mushumba and Jan-Peter Sperhake contributed equally to this work.

This article is part of the Topical Collection on COVID-19

Carolin Edler and Ann Sophie Schröder share first authorship, and Herbert Mushumba and Jan-Peter Sperhake share last authorship

Jan-Peter Sperhake

sperhake@uke.de

1 Department of Legal Medicine, University Medical Center Hamburg-Eppendorf, Butenfeld 34, 22529 Hamburg, Germany

2 Department of Pathology, University Medical Center Hamburg-Eppendorf, Hamburg, Germany

3 Institute of Microbiology, University Medical Center Hamburg-Eppendorf, Hamburg, Germany

\section{Introduction}

Autopsies of deceased with a confirmed severe acute respiratory syndrome coronavirus 2 (SARS-CoV-2) infection can provide important insights into the novel disease and its course. Furthermore, autopsies are essential for the correct statistical recording of the coronavirus disease 2019 (COVID-19) deaths. Contrary to the initial recommendation of the German Robert Koch Institute (RKI) to avoid autopsies of COVID-19 deaths if possible [1], this institution has recently changed its recommendation and currently acknowledges the benefits and value of autopsies in the context of pandemic control.

In the northern German Federal State of Hamburg, which has a population of about 1.8 million, all deaths of Hamburg 
citizens with confirmed SARS-CoV-2 infection have been examined by postmortem computer tomography (PMCT) and autopsied at the Department of Legal Medicine (DLM) of the University Medical Center Hamburg-Eppendorf (UKE) since the pandemic outbreak in Germany. With the exception of the first case (a man who died outside of Europe), the autopsy orders were issued by the Hamburg public health authorities in accordance with \$25(4) of the German Infection Protection Act (Gesetz zur Verhütung und Bekämpfung von Infektionskrankheiten beim Menschen). To our knowledge, this approach is unique in the Federal Republic of Germany. There is no information in the English language literature on a comparable existing procedure elsewhere in the world. In Hamburg, both deceased from the outpatient sector (nursing and old people's homes, domesticity) and deceased from the hospital are autopsied. The SARS-CoV-2 infections were diagnosed either antemortem or postmortem in the mortuary (DLM or crematorium). The postmortem detection of SARS-CoV-2 can be done by a nasopharyngeal swab analogous to the tests in living persons or by lung biopsies. The study cohort thus also includes deceased in whom no SARS-CoV-2 infection was known antemortem, hence highlighting a part of the dark field. Taking into account the medical history, the PMCT, and autopsy findings, it could finally be determined whether the SARS-CoV-2 infection was the cause of death or whether death occurred independently of the virus infection. Death is classified as SARS-CoV-2 infection-related if the infection at least contributed to the death, according to categories $1-3$, the definition of which is explained in the Methods section. The cause of death is determined on autopsy and the assessment of whether or not the death is SARSCoV-2-related is passed on to the public health authority. The result is then included in the Hamburg register of death statistics.

A SARS-CoV-2-infected corpse is most likely contagious. The Committee for Biological Agents (ABAS) has classified SARS-CoV-2 in risk group 3 [2]. However, in the case of an autopsy with appropriate protective measures, an increased risk of transmission is not to be expected for the personnel [3]. None of the forensic pathologists or medical staff that were involved in the autopsies of this study (and subsequent ones) showed COVID-19 disease symptoms at the time of writing this manuscript.

The so-called minimally invasive autopsies are only used to obtain selective findings. The individual pathomorphologic picture and cause of death cannot be fully determined. Therefore, SARS-CoV-2-infected bodies in Hamburg are always completely autopsied (opening of all three body cavities and dissection of all organs, including a dissection of the veins of the lower extremities). The autopsies are carried out by at least one forensic specialist and a resident. This ensures that the autopsies meet the standards for forensic autopsies.
Systematic registration of all COVID-19 autopsy cases in the German-speaking world has just started [4]. The current content of this register, however, is not publicly available. Currently, there is only one publication in the English language literature of two complete autopsies of deceased with a SARS-CoV-2 infection in the USA [5].

Our evaluation provides a systematic overview of the first 80 consecutive full autopsies of deceased with confirmed SARS-CoV-2 infection in Hamburg, Germany.

\section{Methods}

The first consecutive 80 autopsies of persons who tested positive for SARS-CoV-2 ante- or postmortem, performed at the DLM between March 20 and April 18, 2020, were evaluated. All documents available at the DLM concerning the deaths (medical history, medical records, police investigation reports, death certificate, PMCT, and autopsy protocols) were evaluated descriptively. Complete results of additional investigations (e.g. histology, virology, and neuropathological examinations of the brain) are so far only partially available and are therefore not the main focus of the present evaluation.

A categorisation of deaths with SARS-CoV-2 infection is proposed in this study (Table 1). The corresponding categorisation of the 80 cases presented was carried out independently by two forensic specialists.

Quantitative SARS-CoV-2 RNA RT-PCR was performed from swabs as previously described by Pfefferle et al. [6].

A subset of the 80 cases included in the study has already been used in other studies that are already published or currently under review. This concerns case 1 (Heinrich et al.), cases 2-13 [7], and cases 2-13, 15, 19-23, 27-29, 33, 3638,40 , and 41 [8].

\section{Results}

Of the examined 80 deceased with SARS-CoV-2 infection, all but the first autopsy, which had been requested by relatives, were ordered by the public health department. The autopsies were performed on average 4 days after death.

The deceased were aged between 52 and 96 years (average 79.2 years, median 82.4 years). Thirty-four of the deceased were female (42\%) and 46 male (58\%). In twelve cases, the place of death was the patient's own home (15\%); in 51 cases, the hospital (64\%); in thirteen cases, a nursing or retirement home (16\%); and in one case, a hotel. In three cases, no information was available on the place of death. Of the patients who died in hospitals, 17 died in intensive care units (ICU) with invasive ventilation, 31 in a normal ward, and one in the emergency room. The exact place of death could not be determined with regard to two patients. 
Table 1 Categorisation of SARS-CoV-2-positive deaths

\begin{tabular}{|c|c|}
\hline Category & Explanation \\
\hline Category 1: Definite COVID-19 death & Autoptic pneumonia and/or ARDS as cause of death \\
\hline Category 2: Probable COVID-19 death & $\begin{array}{l}\text { Autoptic pneumonia and/or ARDS and other infectious causes of death } \\
\text { (e.g. pulmonary embolism) }\end{array}$ \\
\hline $\begin{array}{l}\text { Category 3: Possible COVID-19 } \\
\text { death with an equal alternative cause of death }\end{array}$ & $\begin{array}{l}\text { Cause of death that cannot be determined with certainty by autopsy } \\
\text { (e.g. cardiac arrhythmia in cardiomyopathy) } \\
\text { OR } \\
\text { autoptic respiratory tract infection/pneumonia of other genesis } \\
\text { (e.g. aspiration pneumonia, exacerbated COPD) }\end{array}$ \\
\hline $\begin{array}{l}\text { Category 4: SARS-CoV-2 } \\
\text { detection with cause of death not associated to COVID-19 }\end{array}$ & $\begin{array}{l}\text { Clear non-SARS-CoV-2-related cause of death } \\
\text { (e.g. brain mass haemorrhage in hypertension, } \\
\text { acute myocardial infarction in coronary thrombosis) }\end{array}$ \\
\hline
\end{tabular}

The average body mass index (BMI) was $25.9 \mathrm{~kg} / \mathrm{m}^{2}$. Overall, $38 \%$ of the deceased were overweight or obese (overweight 13 cases, obesity grade 1 six cases, grade 2 five cases, grade 3 six cases).

All deceased, except for two women, in whom no significant pre-existing conditions were seen autoptically (cases 7 and 50), had relevant previous illnesses. The vast majority had diseases of the cardiovascular system (85\%), followed by lung diseases (55\%), kidney diseases (34\%), and central nervous system (CNS) diseases (35\%). Diabetes mellitus was known in $21 \%$ of the deceased, and carcinomas/ haematological diseases in $16 \%$. Table 2 gives a systematic overview of all the cases.

In six of the deceased, SARS-CoV-2 infection was diagnosed postmortem. In the other 74 cases, SARS-CoV-2 infection had already been known antemortem. The time of the first positive PCR test is known in 49 cases. The average survival time after the first positive test until death was 6 days. The longest documented survival time was 32 days in the case of an 89-year-old man who died in a normal hospital ward.

In most cases, the infection pathway could only be speculated. For example, 25 of the deceased came from nursing homes or residential and care facilities for the disabled, in which other residents had been diagnosed with SARS-CoV2 infections previously. In two cases, the infection was presumably transmitted in the hospital by fellow patients; in six cases, the infection was probably caused by travel to countries defined as risk areas at that time; and in one case, presumably by contact with family members at a family celebration.

A total of 76 cases (95\%) were classified as COVID-19 deaths, corresponding to categories $1-3$. Four deaths (5\%) were unrelated to SARS-CoV-2 infection. In these four deaths, autopsy and radiological findings suggestive of COVID-19 were missing. The causes of death in these four cases were pericardial tamponade as a complication of myocardial infarction (case 39), sepsis secondary to necrotizing fasciitis (case 48), and two sudden cardiac deaths due to severe heart disease (CHD, dilated cardiomyopathy; cases 23 and 32). In three of these four cases, SARS-CoV-2 infection had been diagnosed postmortem by nasopharyngeal swabs. Typical COVID-19 symptoms such as cough, sore throat, impaired taste or smell, or flu-like infection were not known in any of the four cases antemortem. However, one of the deceased (case 23) was found to have an elevated body temperature postmortem.

Fifty-seven cases $(71 \%)$ corresponded to category 1 . In all these cases, pneumonia, with or without evidence of sepsis, was found to be the cause of death. Also in category 2, pneumonia was present in all 10 cases (25\%). In seven of these 10 cases, however, a fulminant pulmonary artery embolism was fatal, and in one case each, aortic valve endocarditis, septic encephalopathy, and hepatorenal failure secondary to liver cirrhosis were contributory causes of death. A total of eight cases $(10 \%)$ were classified in category 3 in which a competing cause of death is also considered in addition to COVID-19 (e.g. aspiration pneumonia, pronounced emphysema without evidence of pneumonia, or acute bronchitis). In these cases, a relation with SARS-CoV-2 infection can certainly be discussed critically.

In addition to the eight fatal fulminant pulmonary artery embolisms, peripheral pulmonary artery embolisms were found in nine other cases - a total of 17 cases (21\%) altogether. In each of these deaths as well as in fifteen others (in total 32 cases, 40\%), thrombi were found in the deep veins of the lower extremities. Of these deaths, 21 were male and 11 female (ratio 1.9:1). The male deceased also showed thrombi in the prostatic venous plexus in 15 cases and in the veins of the oesophagus in one case. The average BMI of these 32 deceased was $28.5 \mathrm{~kg} / \mathrm{m}^{2}$. The places of death were hospitals (12 cases in the ICU, seven cases in the normal ward, one case in the emergency room), nursing homes (six cases), and own homes (four cases).

For the first 30 deceased autopsied, combined naso- and oropharyngeal swabs and swabs of the lung tissue were taken at the time of the dissection. In all these 30 cases, a SARS$\mathrm{CoV}-2$ infection could be diagnosed by PCR postmortem. The maximum PMI in these cases was 12 days. 


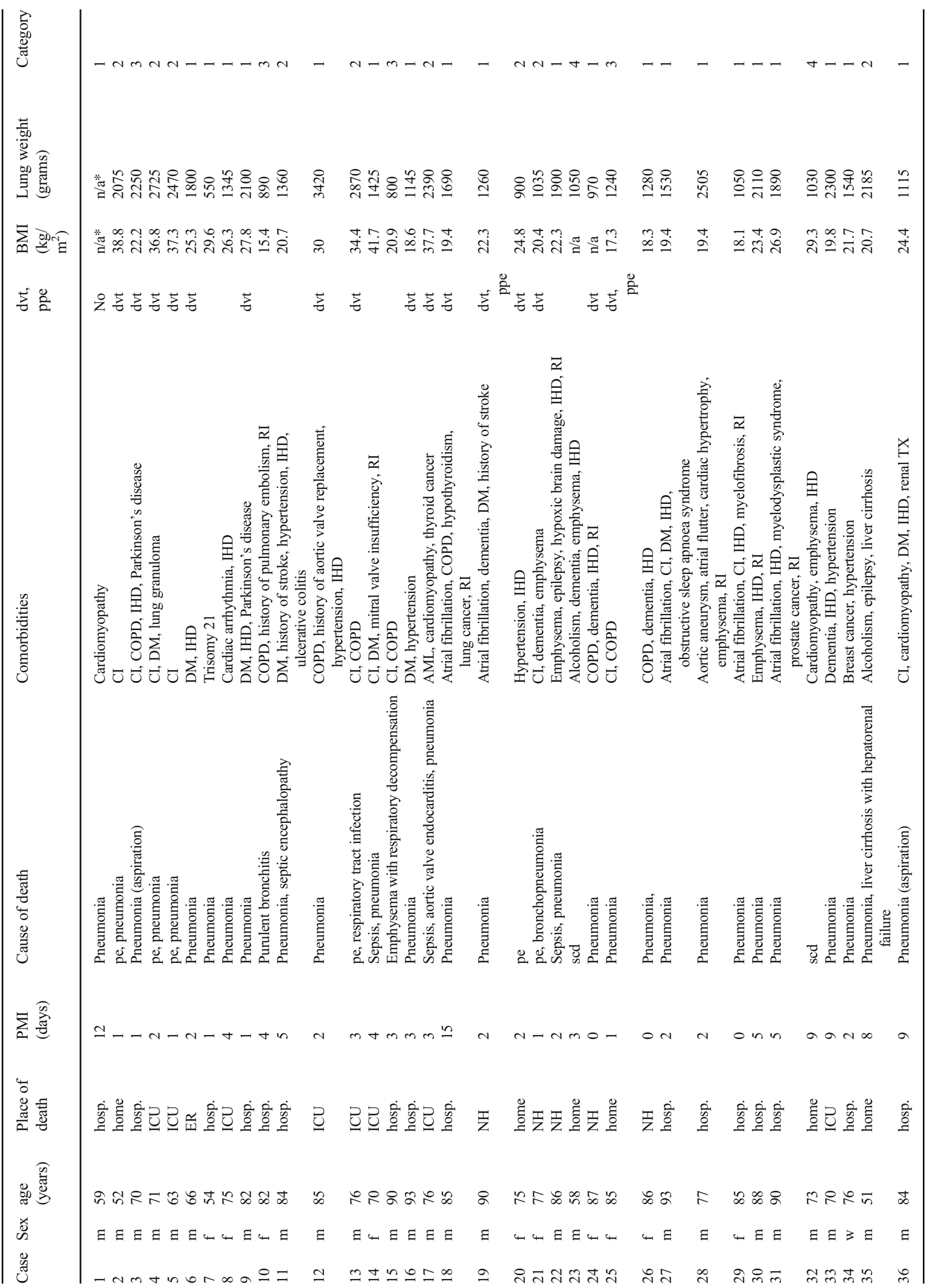




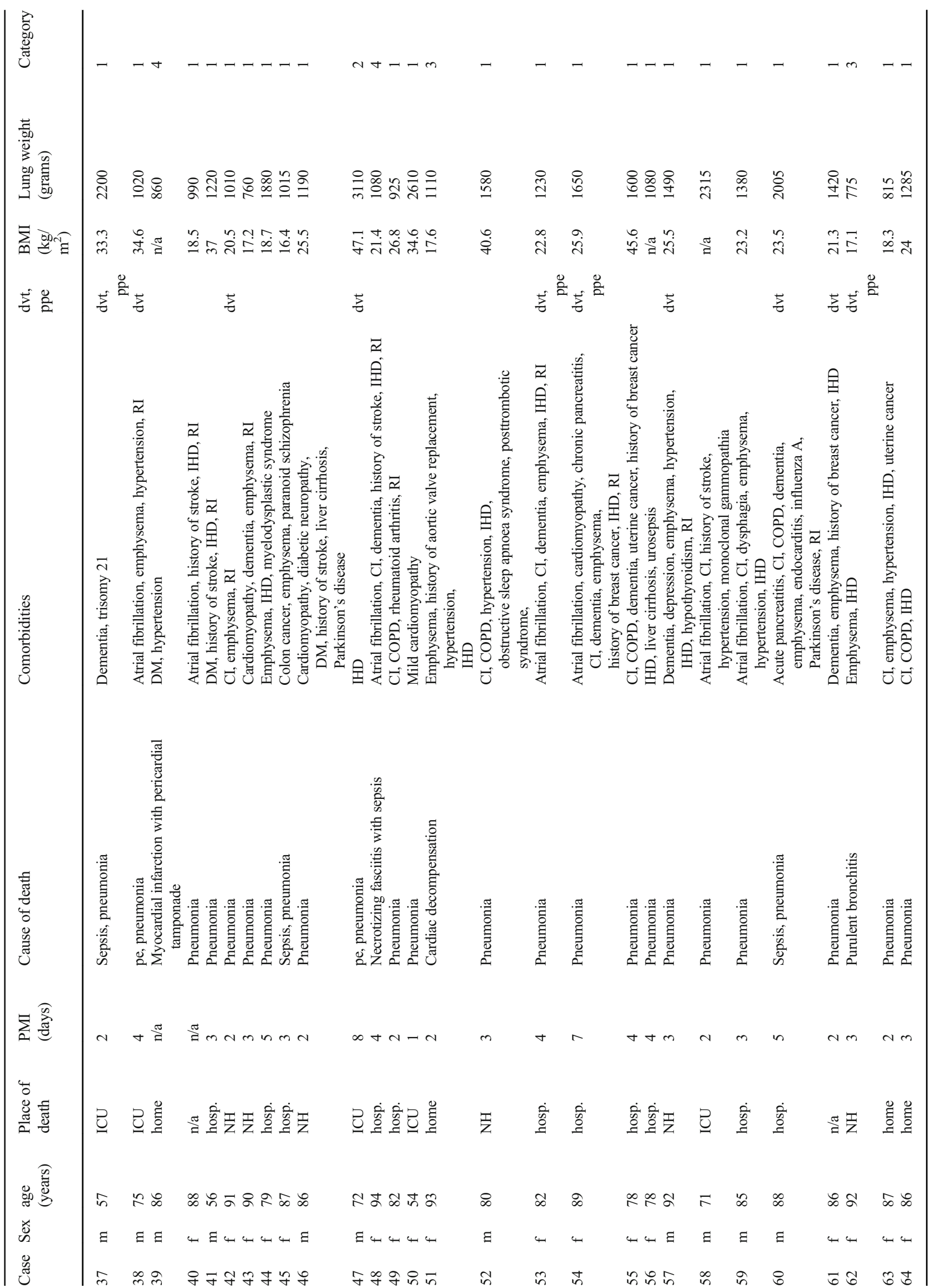




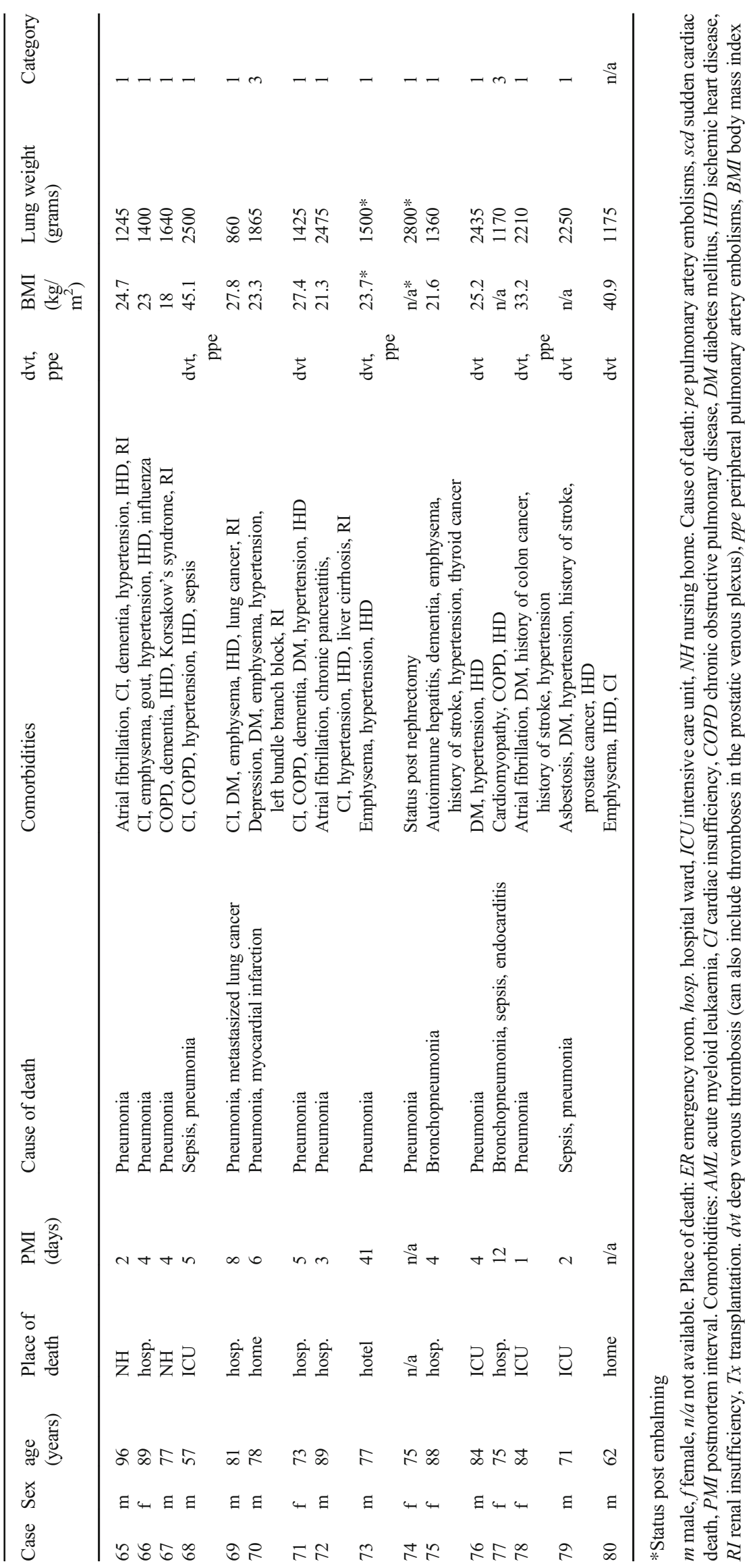


Fig. 1 a Heavy, congested lungs (case 52). b Patchy pleural surface with segmental hyperemia (case 2). c Cutting surface of the lung with alternating hyperemic and pale areas (case 5). d Lung surface with pleurisy (case 12). e Thrombosis of the deep veins of the lower extremity (case 4). $\mathbf{f}$ Pulmonary embolism (case 4)

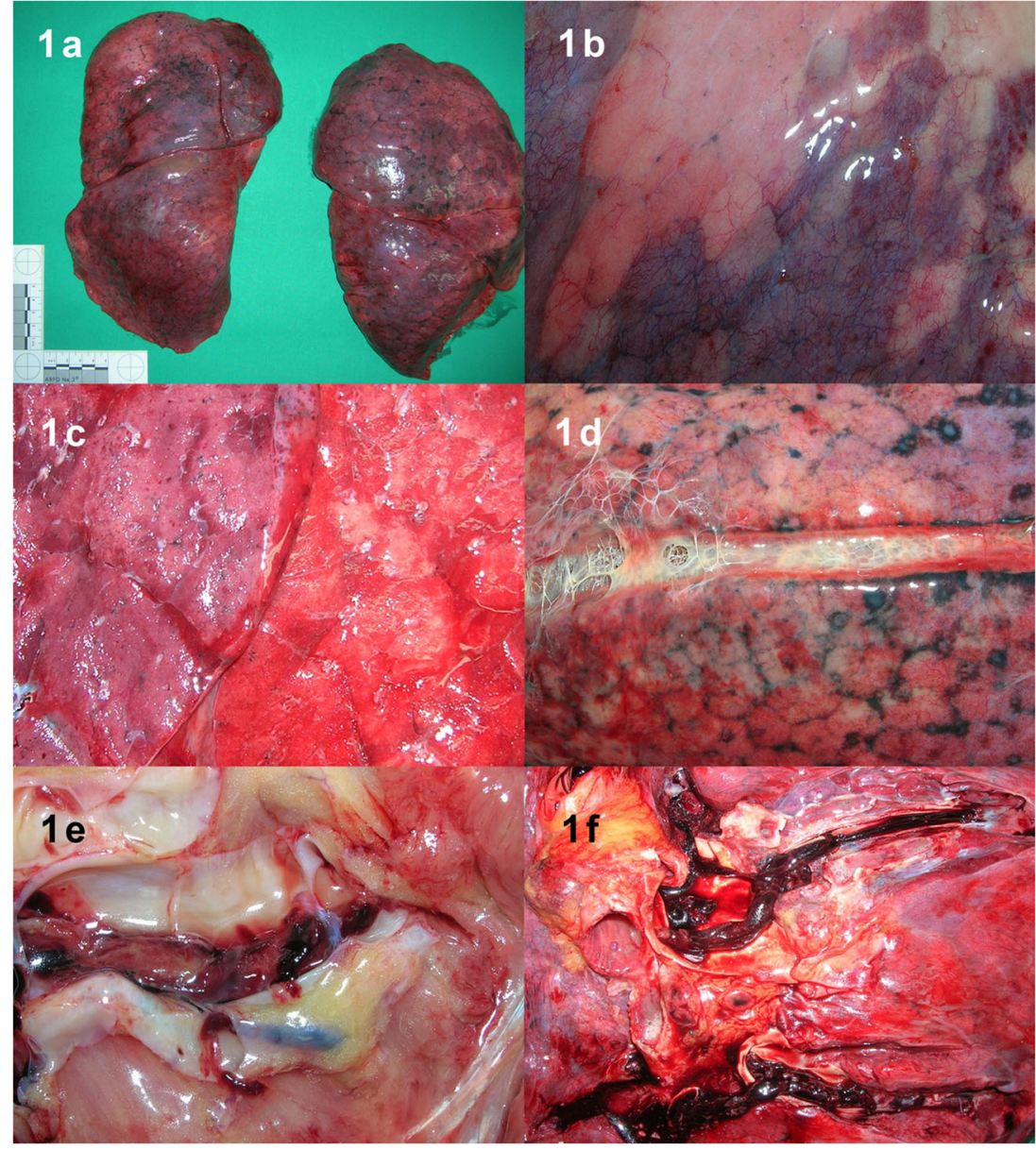

Macroscopic lung findings revealed a broad spectrum of changes, often overlaid by chronic diseases such as chronic bronchitis and emphysema. It seems to be typical for COVID-19 pneumonia that the lungs are very large and heavy due to retained fluid (Fig. 1a). The mean combined lung weight was $1,610 \mathrm{~g}$. Standard combined lung weights are $639 \mathrm{~g}$ (female) and $840 \mathrm{~g}$ (male) $[9,10]$. The lung surfaces sometimes showed signs of pleurisy (Fig. 1d). Typically, a mosaic-like pattern of pale fields and slightly protruding dark purple sections with prominent capillary drawing has been seen (Fig. 1b). On the cut surfaces, the affected lung sections were either ubiquitously dark red or also alternately faded (Fig. 1c). The tissue was diffusely solidified but at the same time fragile. However, in some of the cases, lung changes seen in COVID-19associated deaths appeared as a purulent respiratory tract infection with abscessed bronchopneumonia. In these cases, the typical macroscopic signs of acute respiratory distress syndrome were not very pronounced or were absent altogether.

Histologically, 8 cases (only the first 12 cases have been evaluated so far) showed diffuse alveolar damage (DAD) with activated type II pneumocytes, fibroblasts, protein-rich exudate, and hyaline membranes (Fig. 2a and d). In advanced stages, squamous metaplasia and fibrosis occurred (Fig. 2b).
In some cases, giant cells and megakaryocytes appeared. The small pulmonary arteries often showed a pronounced infiltrate of lymphocytes and plasma cells, whereby the endothelia were not reactively altered in the sense of vasculitis (Fig. 2c). However, there were four cases in which the picture of a granulocyte-dominated focal confluent bronchopneumonia was dominant (Fig. 2e). In these cases, there was often chronic, purulent exacerbated bronchitis. Mixed forms of DAD and purulent pneumonia also occurred in different stages of organisation. Some patients had advanced stages of emphysema with destruction of alveolar septae, fibrosis, and lymphocytic infiltrates.

Other organs often showed signs of chronic diseases, such as scarring in the myocardium, arterio-arteriolosclerosis of the kidneys, and congestion of the liver and spleen. A small lymphocytic infiltrate in the right ventricle of the heart was present in one case as a sign of myocarditis (case 4).

Mild to pronounced lymphocytic pharyngitis was found in 7 of the 8 cases examined (Fig. 2f). Shock changes in the liver, kidneys, or intestine were found in half of the cases. The veins of the lower extremities showed no pathological changes, except for varying degrees of phlebosclerosis and fresh thrombi, and in particular, no signs of phlebitis. 
Fig. 2 (all H\&E) a Lung: diffuse alveolar damage (case $2, \times 80$ ). b Lung: squamous metaplasia (case $4, \times 50$ ). c Lymphocytes in the wall of a small pulmonary artery (case $2, \times 80$ ). d Lung: hyaline membranes (case $5, \times 80$ ). e Purulent pneumonia (case $7, \times$ 80). f Pharyngitis with predominant lymphoplasmacellular infiltrate (case $11, \times 80$ )
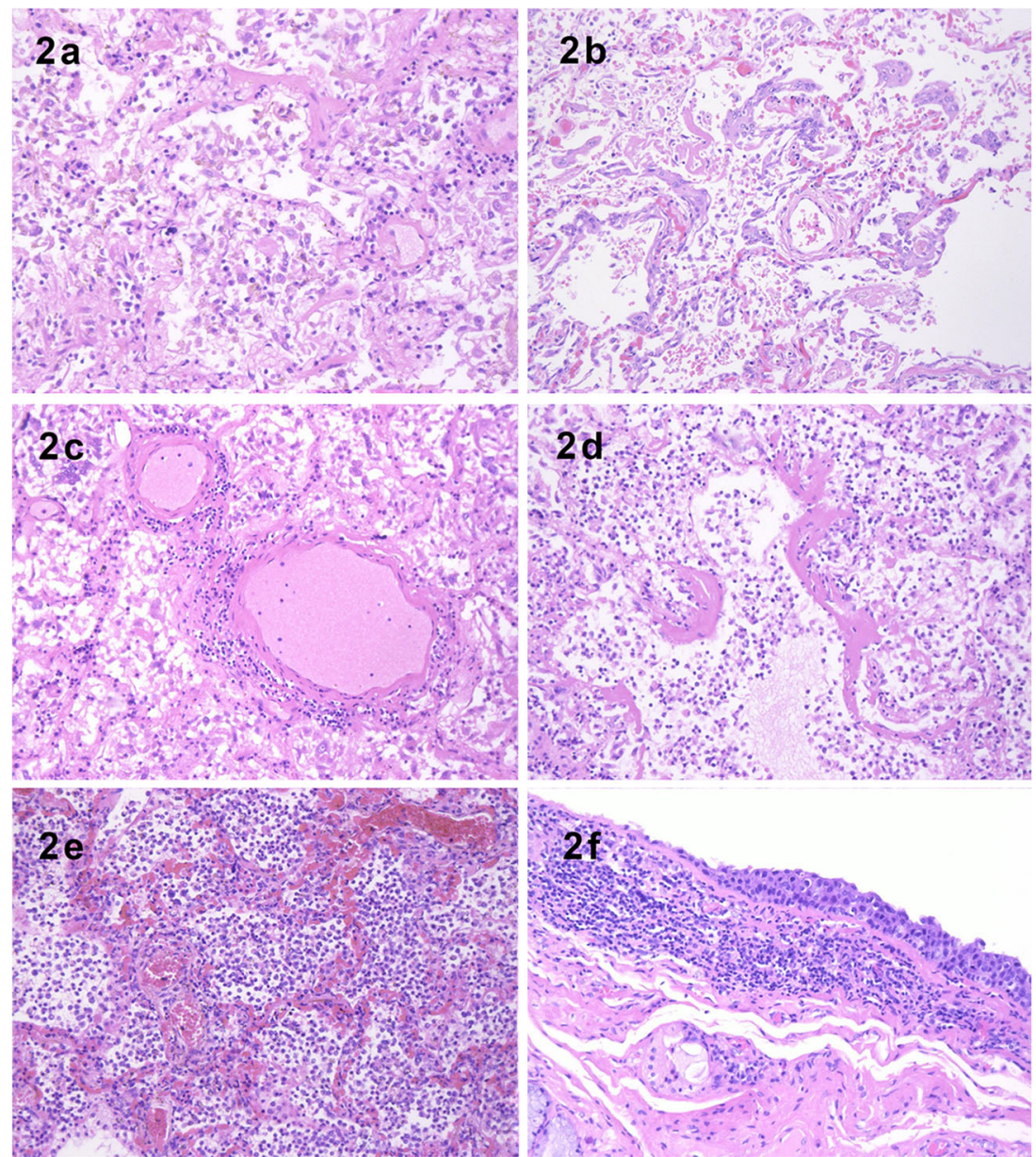

\section{Discussion}

The study provides an overview of 80 autopsies on SARSCoV-2-infected persons in the German city of Hamburg. To our knowledge, this is the largest overview of autopsies of SARS-CoV-2-infected persons presented to date.

An autopsy is indispensable to clarify the cause of death as well as for quality control. The discrepancy between the clinically determined cause of death and autopsy results is well described $[11,12]$. The autopsy provides essential information on the pathology of COVID-19. By tissue sampling, it can provide preliminary answers to questions such as where in the body the virus replicates and whether organs other than the lungs and throat are affected.

The average age of the deceased was 79.2 years (median 82.4 years) and none of the deceased was younger than 52 years. According to the RKI, however, most infections are found between 15 and 59 years of age (68\%). The age distribution as well as the gender ratio of men to women of 1.35:1 in the presented cohort corresponds to the data collected nationwide in Germany by the RKI [13].

With the exception of two cases, all the deceased suffered from severe pre-existing conditions, predominantly of the cardiovascular system and the lungs. About $21 \%$ of the deceased showed obesity (BMI $>30 \mathrm{~kg} / \mathrm{m}^{2}$ ); the average BMI was $25.9 \mathrm{~kg} / \mathrm{m}^{2}$. This corresponds to the figures published by the RKI for the occurrence of obesity in the general population in Germany [14]. However, in the group of deceased persons who had developed thrombi and possibly a pulmonary artery embolism, the average BMI was higher at $28.5 \mathrm{~kg} / \mathrm{m}^{2}$.

Several studies have already reported that pulmonary embolism and coagulopathy are frequent in patients with COVID-19 [15-17]. Varga et al. also reported inflammatory reactions of the endothelium in various organs (lung, heart, small intestine) [18], a finding that was not reproducible in our histologically examined cases. In the cohort presented, pulmonary embolism and thrombosis occurred in hospitalized patients as well as in patients who died in an outpatient or domestic setting. Thrombi in the deep veins of the lower legs were found in almost every second male, but only in about every third female.

The most frequent cause of death was pneumonia, followed by pulmonary artery embolisms combined with pneumonia. Overall, COVID-19 pneumonia was found in $83 \%$ of the deceased. Most of these were virus-induced lung changes in the sense of diffuse alveolar damage. However, bacterial 
superinfected bronchopneumonia also occurred (no bacteriological diagnosis was made postmortem). In $11 \%$ of the deaths, competing causes of death were considered. In 5\%, there were clear causes of death not related to SARS-CoV-2 infection. Failure to perform postmortem examinations erroneously includes category 4 cases in the statistics of corona deaths. A higher mortality rate is the result. As a result of the postmortem examinations, the four cases were not included in the statistics of COVID-19 deaths in Hamburg. Among these, three were sudden deaths in an outpatient setting while one occurred in a hospital. From an epidemiological point of view, the question of whether a person died with or as a result of SARS-CoV-2 might be rather secondary. Nevertheless, the answer to this question may be important in individual cases not only for the relatives of those affected but also for the general population especially, if it is assumed that a young person or a (supposedly) healthy person suddenly died of SARS-CoV-2.

Postmortem evidence of SARS-CoV-2 infection by means of a naso- or oropharyngeal swabs was found in all 30 cases tested, up to a maximum PMI of 12 days. Thus, postmortem diagnosis should be unproblematic in the daily autopsy routine. In the meantime, the DLM has been able to detect a SARS$\mathrm{CoV}-2$ infection even in an advanced decomposed corpse.

We are convinced that autopsies of COVID-19 deceased patients can make an invaluable contribution that goes beyond the recognition of individual causes of death. Not least, as a result of the autopsy observations, the treatment regimen for COVID-19 patients in Hamburg has already been adapted with a view towards more effective anticoagulation. Autopsies and targeted tissue preservation open up the field for a wide range of scientific activities, which in the future may be key to both understanding the disease and innovative therapeutic approaches.

\section{Compliance with ethical standards}

Conflict of interest The authors declare that they have no conflict of interest.

Ethical approval All procedures performed in studies involving human participants were in accordance with ethical standards and with the 1964 Helsinki declaration and its later amendments or comparable ethical standards. In accordance with the Infection Protection Act and the Hamburg Section Act, autopsy results including histology may be used anonymously for scientific evaluation.

\section{References}

1. Robert Koch Institute (2020) Empfehlungen zum Umgang mit SARS-Cov-2-infizierten Verstorbenen. https:/www.rki.de/DE/ Content/InfAZ/N/Neuartiges_Coronavirus/ Verstorbene.html. Accessed 16 March 2020
2. The Committee for Biological Agents (2020) Einstufung des SARS-CoV-2 in Risikogruppe 3 und Empfehlungen zur Labordiagnostik https://www.baua.de/DE/Aufgaben/ Geschaeftsfuehrung-von-Ausschuessen/ABAS/pdf/SARS-CoV-2. pdf? blob=publicationFile\&v=3. Accessed 20 April 2020

3. DGRM (2020) Joint statement of the German Society for Forensic Medicine and the Professional Association of German Forensic Doctors on the handling of deceased and living persons infected with SARS-CoV-2 in forensic medicine. https://www.dgrm.de/ fileadmin/PDF/PDF Duesseldorf/Stellungnahme DGRM Berufsverband_zu_Umgang_mit_COVID_19.pdf. Accessed 20 April 2020

4. DeRegCOVID (2020) Deutsches Register von COVID-19 Obduzierten Fällen. https://www.pathologie.de/?eID= downloadtool\&uid=1994. Accessed 20 April 2020

5. Barton LM, Duval EJ, Stroberg E, Ghosh S, Mukhopadhyay S (2020) COVID-19 autopsies, Oklahoma, USA. Am J Clin Pathol 153:725-733. https://doi.org/10.1093/ajcp/aqaa062

6. Pfefferle S, Reucher S, Nörz D, Lütgehetmann M (2020) Evaluation of a quantitative RT-PCR assay for the detection of the emerging coronavirus SARS-CoV-2 using a high throughput system. Euro Surveill 25(9). https://doi.org/10.2807/1560-7917. ES.2020.25.9.2000152

7. Wichmann D, Sperhake JP, Lütgehetmann M, Steurer S, Edler C, Heinemann A, Heinrich F, Mushumba H, Kniep I, Schröder AS, Burdelski C, de Heer G, Nierhaus A, Frings D, Pfefferle S, Becker $\mathrm{H}$, Bredereke-Wiedling $\mathrm{H}$, de Weerth A, Paschen HR, Sheikhzadeh-Eggers S, Stang A, Schmiedel S, Bokemeyer C, Addo MM, Aepfelbacher M, Püschel K, Kluge S (2020) Autopsy findings and venous thromboembolism in patients with COVID-19 - a prospective cohort study. Ann Intern Med. https://doi.org/10. 7326/M20-2003

8. Puelles VG, Lütgehetmann M, Lindenmeyer MT, Sperhake JP, Wong MN, Allweiss L, Chilla S, Heinemann A, Wanner N, Liu S, Braun F, Lu S, Pfefferle S, Schröder AS, Edler C, Gross O, Glatzel M, Wichmann D, Wiech T, Kluge S, Pueschel K, Aepfelbacher M, Huber TB (2020) Multi-organ and renal tropism of SARS-CoV-2. NEJM. https://doi.org/10.1056/NEJMc2011400

9. Molina DK, DiMaio VJ (2012) Normal organ weights in men: part II-the brain, lungs, liver, spleen, and kidneys. Am J Forensic Med Pathol 33(4):368-372

10. Molina DK, DiMaio VJ (2015) Normal organ weights in men: part II-the brain, lungs, liver, spleen, and kidneys. Am J Forensic Med Pathol 36(3):182-187

11. Schönamsgruber N, Schröder C, Edler C, Püschel K, Sperhake JP, Schröder AS (2019) Quality of external post-mortem examination and quality of death certificates at the University Hospital in Hamburg. Rechtsmedizin 29:281-286

12. Ermenc B (2000) Comparison of the clinical and post mortem diagnoses of the cause of death. Forensic Sci Int 114:117-119

13. Robert Koch Institute (2020) https://www.rki.de/DE/Content/ InfAZ/N/Neuartiges_Coronavirus/Situationsberichte/2020-04-18de.pdf? Accessed 20 April 2020

14. Robert Koch Institute (2020) https://www.rki.de/DE/Content/ Gesundheitsmonitoring/Themen/Uebergewicht Adipositas/ Uebergewicht_Adipositas_node.html. Accessed 20 April 2020

15. Giannis D, Ziogas AI, Giannic P (2020) Coagulation disorders in coronavirus infected patients: COVID-19, SARS-CoV-1, MERS$\mathrm{CoV}$ and lessons from the past. J Clin Virol:j.jcv.2020.104362

16. Guan W, Ni Z, Hu Y, Liang C et al (2020) Clinical characteristics of coronavirus disease 2019 in China. N Engl J Med 382:1708-1720. https://doi.org/10.1016/NEJMoa2002032

17. Han H, Yang L, Liu R, Liu F, Wu KL, Li J, Liu XH, Zhu CL (2020) Prominent changes in blood coagulation of patients with SARSCoV-2 infection. Clin Chem Lab Med 0. https://doi.org/10.1515/ cclm-2020-0188 
18. Varga Z, Flammer AJ, Steiger P, Haberecker M, Andermatt R, Zinkernagel AS, Mehra MR, Schuepbach RA, Ruschitzka F, Moch H (2020) Endothelial cell infection and endotheliitis in COVID-19. Lancet. 395:1417-1418. https://doi.org/10.1016/ S0140-6736(20)30937-5
Publisher's note Springer Nature remains neutral with regard to jurisdictional claims in published maps and institutional affiliations. 